\title{
Le soutien documentaire au réseau québécois de santé publique
}

\section{Information services for the public health network in Québec El apoyo documental a la red quebequense de la salud pública}

\section{Sylvie Desbiens}

Volume 41, numéro 2, avril-juin 1995

Les bibliothèques de la santé

URI : https://id.erudit.org/iderudit/1033302ar

DOI : https://doi.org/10.7202/1033302ar

Aller au sommaire du numéro

Éditeur(s)

Association pour l'avancement des sciences et des techniques de la documentation (ASTED)

ISSN

0315-2340 (imprimé)

2291-8949 (numérique)

Découvrir la revue

Citer cet article

Desbiens, S. (1995). Le soutien documentaire au réseau québécois de santé publique. Documentation et bibliothèques, 41(2), 123-128.

https://doi.org/10.7202/1033302ar
Résumé de l'article

Qu'entend-on par santé publique ? Quel est le champ de pratique dévolu à la santé publique au sein du réseau de la santé au Québec ? Quelles en sont les composantes structurelles et organisationnelles ? De quelle façon la récente réforme menée par le ministère de la Santé affecte-t-elle ce réseau et ses interventions ? L'auteure tente ici de répondre à ces questions tout en développant la spécificité du soutien documentaire requis par cette composante majeure du système de santé québécois. La multidisciplinarité et l'intersectorisation y sont évoquées afin de rendre compte de la complexité et de l'étendue du champ documentaire à couvrir par les documentalistes de ce secteur d'activité. Enfin, l'auteure présente de façon plus spécifique la banque de données SANTÉCOM, spécialisée en santé publique.
Tous droits réservés (c) Association pour l'avancement des sciences et des techniques de la documentation (ASTED), 1995
Ce document est protégé par la loi sur le droit d'auteur. L’utilisation des services d'Érudit (y compris la reproduction) est assujettie à sa politique d'utilisation que vous pouvez consulter en ligne.

https://apropos.erudit.org/fr/usagers/politique-dutilisation/ 


\title{
Le soutien documentaire au réseau québécois de santé publique
}

\author{
Sylvie Desbiens \\ Bibliothécaire, Direction régionale de la Santé publique de la Montérégie
}

Qu'entend-on par santé publique? Quel est le champ de pratique dévolu à la santé publique au sein du réseau de la santé au Québec? Quelles en sont les composantes structurelles et organisationnelles? De quelle façon la récente réforme menée par le ministère de la Santé affecte-t-elle ce réseau et ses interventions? L'auteure tente ici de répondre à ces questions tout en développant la spécificité du soutien documentaire requis parcette composante majeure du système de santé québécois. La multidisciplinarité etl'intersectorisation y sont évoquées afin de rendre compte de la complexité et de l'étendue du champ documentaire à couvrir par les documentalistes de ce secteur d'activité. Enfin, l'auteure présente de façon plus spécifique la banque de données SANTÉCOM, spécialisée en santé publique.

Information services for the public health network in Québec

What is public health? How does public health fit into the Québec health network? How is public health strutured and organised? How does the recent reform of health services carried out by the ministère de la Santé affect public health? In this article, the author answers these questions and develops the specificity of library services required by this large component of the Québec health system. Public health is a multidisciplinary activity and the collections reflect this aspect. She also describes SANTÉCOM, a public health data base.

\section{El apoyo documental a la red quebequense de la salud pública}

¿Qué queremos decir por salud pública? ¿Cual es el campo de práctica atribuido a la salud pública en el seno de la red de la salud en la provincia de Quebec? ¿Cuales son sus partes componentes estructurales y organizacionales? ¿De que manera la reforma reciente dirigida por el Ministerio de la Salud influye en esta red y en sus intervenciones? La autora trata aqui de responder estas preguntas al mismo tiempo que desarrolla la especificidad del apoyo documental requerido por esta parte componente importante del sistema de salud quebequense. La multidisciplinaridad y la intersectorialidad son evocadas aquí con el propósito de dar cuenta de la complejidad y de la amplitud del campo documental a cubrir por los documentalistas de este sector de actividad. Por fin, la autora presenta, de manera más especifica, el banco de datos SANTÉCOM, especializado en la salud pública.
Quelques considérations sur la santé communautaire et la santé publique au Québec depuis 1970: évolution et organisation

Au début des années 1970, dans le prolongement de la Commission Castonguay-Nepveu et de la réforme du système de santé, une nouvelle approche s'imposa avec de plus en plus de force: la santé communautaire. En 1977, le docteur Jean Rochon (actuel ministre de la Santé) définissait la santé communautaire en ces termes: "La santé communautaire peut se définir comme l'art et la science d'améliorerl'état de santé de la population, de prévenir la maladie et de promouvoir l'efficacité des services de santé par la coordination des efforts communautaires». Cette approche était stimulée par des considérations d'efficacité, d'éthique, de coûts sociaux et d'équité. Elle est d'ailleurs encore d'actualité puisqu'inscrite au premier plan de la plus récente réforme du système de santé québécois (1990) et de la première politique officielle de santé au Québec (1993).

Les départements de santé communautaire (DSC) sont nés en 1973. Ils reçurent comme première responsabilité la planification sanitaire. Cette tâche incluait, à partir de 32 territoires découpés sur la base d'environ 200000 personnes, l'analyse de la situation sanitaire, la détermination des priorités et des objectifs, l'élaboration de stratégies de développement des programmes, leur implantation et leur évaluation. Après avoir reçu le mandat et mis sur pied les services en santé et sécurité du travail en 1980 , ils sont demeurés depuis 1983 responsables de deux mandats principaux: la planification sanitaire et le contrôle des épidémies (incluant les MTS et le SIDA). De 1973 à 1993, année où l'on décrétaitl'abolition des DSC, la gestion de la santé publique québécoise s'est donc organisée essentiellement autour des 32 départements de santé communautaire.

En parallèle, les universités renforçaient la formation professionnelle en créant des départements de médecine sociale et préventive aptes à décerner des diplômes de maîtrise ou de spécialisation en santé communautaire. Les chefs de DSC devaient obligatoirement être reconnus comme médecins spécialistes pour occuper cette fonction. Même si la 
pratique des DSC au cours des vingt dernières années a généré des impacts positifs sur la santé publique, les différents intervenants du réseau ainsi que le ministère de la Santé convenaient au début de la présente décennie de procéder à quelques ajustements organisationnels pour faire face aux nouveaux défis.

Aussi, suite à la réforme de 1990, le réseau de santé publique s'articule dorénavant autour des Directions de la santé publique des 16 Régies régionales de la santé et des services sociaux, réparties sur l'ensemble du territoire québécois seIon le découpage par régions administratives en vigueur au Québec. Leurs domaines d'intervention incluent les maladies infectieuses (dont les MTS et le SIDA), la santé environnementale, la santé au travail, la promotion de la santé, la prévention (des maladies, accidents, suicides...) et la protection de la santé publique. Ils ont aussi la charge d'informer la population sur son état de santé, les problèmes prioritaires, les facteurs de risque, les groupes à risque et les intervention jugées les plus efficaces.

Les seize Directions régionales de santé publique (DRSP) sont dorénavant les maîtres d'oeuvre officiels de la santé publique au Québec. Toutefois leurs mandats ne sauraient s'actualiser sans les apports de multiples partenaires. Pensons au réseau de l'éducation appelé à promouvoir de saines habitudes de vie auprès des jeunes ou aux municipalités qui appliquent les règles de sécurité dans les transports, les lois anti-tabac, etc. La contribution de tant de ministères et organismes divers indique bien que la santé globale d'une population dépend des efforts de toute la communauté.

\section{Le soutien documentaire requis par le réseau de santé publique}

Toutes ces considérations permettent d'appréhender l'envergure du «territoire» à couvrir par le réseau de santé publique. L'amplitude de ces champs d'intervention impose dès lors un effort particulier aux documentalistes qui oeuvrent dans ce secteur d'activité.

D'une part, par l'origine multidisciplinaire de ses usagers: professionnels de la santé (médecins spécialistes en santé communautaire, médecins généralistes, dentistes, nutritionnistes, infirmières, épidémiologistes, etc.); professionnels des sciences humaines et sociales (sociologues, psychologues, agents de recherche, administrateurs de la santé, etc.); professionnels des sciences pures (hygiénistes industriels, biologistes, ingénieurs, chimistes, toxicologues, etc.) et dans une moindre mesure, étudiants (niveaux collégial et universitaire) et grand public. D'autre part, par ses ramifications obligées dans à peu près toutes les sphères de l'activitè humaine: l'intersectorisation évoquée plus haut.

Les besoins documentaires sont donc à la fois très pointus et très diversifiés, et les défis sont à la mesure de la superficie à couvrir. S'il est vrai qu'on ne peut plus concevoir notre pratique professionnelle sans le rattachement à divers réseaux ou au sein de partenariats plus ou moins consolidés, cet état de fait est une donnée de base de l'expertise que doivent développer les documentalistes du secteur de la santé publique.

\section{Les réseaux formels et informels}

Sans qu'on ait vu l'émergence de réseaux documentaires formellement constitués dans le champ spécifique de la santé publique au cours des dernières années, les liens avec différents réseaux du domaine de la santé sont néanmoins indispensables pour le prêt-entre-bibliothèques au premier chef, comme pour la référence ou la mise à jour et le développement des collections. Ainsi à titre de responsable de SANTÉCOM et de présidente de la section santé de l'ASTED (1991-1994), j'ai tenté de multiplier les points de convergence entre les intervenants et les centres de documentation pour maximiser les contacts de la santé publique avec ses partenaires.

\section{Les partenariats du réseau de la santé}

Ils sont assez nombreux et diversifiés pour que la Section Santé de l'ASTED procède à un inventaire systématique et en dresse un répertoire publié aux éditions de l'ASTED à la fin de 1992. Environ 200 centres de documentation et bibliothèques y sont recensés. Ces partenaires ont été sollicités à nouveau l'année suivante afin de publier le premier catalogue collectif des collections de périodiques détenues par le secteur de la santé au Québec. 5300 titres distincts sont répertoriés au catalogue, couvrant les domaines médicaux et paramédicaux, l'administration, les sciences humaines et sociales, l'éducation, la bibliothéconomie ou l'informatique. Ce catalogue collectif constitue un outil essentiel pour les documentalistes du secteur de la santé. II facilite la gestion du prêt entre bibliothèques, la rationalisation du développement des collections et l'harmonisation éventuelle des politiques et des tarifications. II assure en outre une visibilité accrue de notre réseau auprès de collaborateurs potentiels, tels que les universités, l'Institut canadien pour l'information scientifique et technique (ICIST), la Bibliothèque nationale du Canada ou les entreprises privées offrant des services spécialisés.

Lespartenariats ponctuels extérieurs au réseau québécois de la santé

Les partenaires ponctuels sont les bibliothèques gouvernementales et paragouvernementales, les bibliothèques universitaires et les centres de documentation des différents groupes de recherche associés au secteur de la santé. À l'extérieur du territoire québécois, il convient également de mentionner l'apport indispensable de l'ICIST, de la Bibliothèque nationale du Canada et des différentes bibliothèques fédérales, de même que celui des autres universités canadiennes pour pallier les lacunes des collections québécoises.

Nous devons recourir fréquemment aux publications gouvernementales américaines (CDC, NTIS, etc.) en les commandant directement auprès des organismes auteurs, ou par l'entremise de distributeurs canadiens tels que I'ICIST. Des liens de collaboration se sont tissés également depuis deux ans entre le Québec et l'Europe francophone grâce à SANTÉCOM, par l'intermédiaire du Réseau francophone internationalen promotion de la santé (RÉFIPS) dont il sera question plus loin.

\section{Les partenariats associatifs}

Plusieurs regroupements documentaires co-existent dans le domaine de la santé. Certains sont établis sur une base 
régionale. Mentionnons, à titre d'exemples, l'Association des bibliothèques de la santé de Montréal et le Groupe des bibliothèques de la santé et des services sociaux de la région 04. D'autres sont regroupés sur une base d'affiliation universitaire. Dans cette catégorie nous pouvons citer l'ABSAUM (Université de Montréal), le MMAHLA (Université McGill) et I'ASDESE (Université de Sherbrooke). D'autres encore sont membres d'un réseau d'établissements, comme le Regroupement des régies régionales.

Ces échanges, plus ou moins formels et plus ou moins soutenus dans le temps, favorisent néanmoins l'uniformisation des pratiques, des ententes de réciprocité, le contrôle des coûts et le partage des innovations. Bref, pour reprendre le jargon technocrate en vigueur, nous dirons qu'ils assurent une meilleure adéquation des ressources en vue d'atteindre une plus grande efficience des services. On ne peut que militer pour le maintien de tels, réseaux ou à tout le moins, pour le renforcement de la Section Santé de I'ASTED. Entre autres, parce que celle-ci est appuyée par une infrastructure permanente qui dessert l'ensemble du territoire québécois et tous les types de milieux documentaires. Elle est donc en meilleure position pour défendre et faire progresser les dossiers communs. Une participation plus active, par exemple, en tant que membre du Conseil d'administration de l'association ouvre sur d'autres perspectives.

\section{Les partenariats informels}

Parmi ces partenariats, nous pouvons privilégier les liens tissés à l'intérieur des associations et des regroupements documentaires et les ouvertures sur de nouveaux milieux qu'ils permettent de développer. Sont également importants les échanges et les liens avec des associations, des regroupements et des comités directement liés à notre milieu de travail. Ainsi, par exemple, la participation de la documentaliste de notre Département de santé communautaire aux rencontres du Conseil de la santé communautaire, qui regroupait les 32 directeurs de DSC et 32 professionnels, durant les trois dernières années de son existence, a permis avec une acuité accrue de saisir à la fois les problématiques de l'heure dans toute leur subtilité et les enjeux politiques des diffé- rents dossiers. Ce sont autant d'éléments précieux pour le développement d'une collection, pour la diffusion sélective de l'information (dsi), voire pour la référence générale. La participation à ce Conseil a ainsi permis d'établir des contacts avec les décideurs du réseau et avecles professionnels de tous les horizons, de faire connaître et valoir l'expertise et les préoccupations des documentalistes et de faire la promotion de la banque de données SANTÉCOM.

Il importe enfin de ne pas négliger les contacts quotidiens avec les professionnels que nous desservons et leurs propres réseaux informels: il est souvent fort intéressant de les connaître afin de les utiliser au besoin. Bref, la seule certitude acquise après dix années vécues à l'intérieur du réseau de santé publique comme documentaliste est celle-ci: le champ d'investigation est tellement large qu'il est essentiel de multiplier les antennes si on désire maximiser la pertinence et les retombées de notre travail. Cette façon de concevoir le travail est également garante d'un intérêt soutenu et d'un plaisir sans cesse renouvelé à oeuvrer dans le milieu.

\section{Les centres de documentation: des DSC aux DRSP}

À peu près la moitié des 32 DSC du Québec ont engagé des ressources pour le soutien documentaire de leur organisation. Le tableau d'ensemble des ressources humaines, matérielles et financières consacrées à la fonction documentaire demeure toutefois très varié. En fait, on y dénombre plusieurs petites collections (périodiques, monographies, audiovisuels et autres matériels promotionnels) présentant de multiples recoupements entre elles. Ce portrait, qui tend tout de même à se modifier à la suite du passage à un nouveau mode d'organisation régionale (de 32 DSC à 16 DRSP), demeure néanmoins fidèle à la situation qui prévalait en 1986, au moment de la mise en disponibilité de la banque de données SANTÉCOM.

SANTÉCOM: la banque de données bibliographiques du réseau québécois de santé publique

\section{Présentation générale}

SANTÉCOM est une banque de données bibliographiques, et non factuelles ou quantitatives. Ses références se situent dans le champ de la santé publique: prévention de la maladie, promotion de la santé, protection de la santé publique. Sans être exclusive, la banque conserve une spécificité québécoise. Elle compte actuellement environ 10000 notices bibliographiques réparties ainsi : 8300 signalements de monographies, de ce nombre, 2500 documents proviennent des DSC et des DRSP; le reste est constitué d'articles tirés de périodiques québécois ou canadiens abordant des problématiques de santé publique. Les monographies proviennent du réseau des $\mathrm{DSC} /$ DRSP, des CLSC, du ministère de la Santé et des Services sociaux, de d'autres ministères provinciaux ou fédéraux, des comptes rendus de conférences ou de colloques, des mémoires ainsi que des ouvrages publiés. Le «matériel promotionnel» (audiovisuels, affiches, dépliants, etc.) en santé publique n'a pu y être intégré à ce jour.

L'objectif visé par cet outil documentaire est le recensement systématique des publications émanant du réseau québécois de santé publique et rendant compte des réalisations de santé publique au Québec. Il faut donc comprendre que cette banque de données ne pourrait ni actuellement ni éventuellement remplacer la consultation de banque de données scientifiques internationales.

\section{Contexte et pertinence}

L'intérêt principal de la banque SANTÉCOM réside dans la mise en commun des ressources en vue de l'atteinte d'une plus grande efficacité des services. En effet, on constate généralement que les liens interministériels et interétablissements au sein du réseau de la santé sont mal articulés et que les réalisations d'ordre scientifique ou technique, théorique ou pratique, demeurent méconnues aussi bien des autres intervenants du réseau que des gestionnaires et, bien sûr, du public en général. Cet état de fait contribue d'ailleurs à entretenir le mythe de l'efficacité restreinte des travailleurs de l'État.

C'est en outre ce type de constatation qui présidait en 1981 à l'élaboration de la 
banque de données ÉDUQ au ministère de l'Éducation du Québec. On peut lire dans l'introduction du bulletin analytique:

La nécessité d'ÉDUQ s'est imposée à la suite d'une analyse de la situation de la recherche en éducation au Québec. II est alors apparu que les ressources humaines et financières consacrées à la recherche développement dans ce domaine n'apportaient pas des retombées proportionnelles aux efforts consentis. En matière d'accès aux travaux, on constatait notamment qu'il était plus facile de prendre connaissance des productions américaines ou européennes que de se renseigner sur les recherches réalisées au Québec, méconnues faute d'inventaire adéquat. Pour satisfaire les besoins manifestés, il apparaissait que pareil inventaire devrait pouvoir senvir d'instrument de référence aux chercheurs, gestionnaires de l'éducation, enseignants et étudiants, en plus de faciliter l'accès aux documents euxmêmes.

C'est pour pallier cette lacune déjà observable dès 1985 que fut formulée la proposition de dépôt officiel et de banque de données «réseau» au Comité de coordination de la santé communautaire (CCSC) de l'Association des hôpitaux du Québec (AHQ). En 1987, celui-ci confiait au DSC Charles LeMoyne le mandat de devenir le dépositaire officiel des publications du réseau des DSC pour alimenter la banque de données. Ce dépôt officiel (et non légal) est toujours en vigueur. II implique l'envoi d'un exemplaire de chaque document produit par un DSC ou une DRSP à SANTÉCOM, pour en assurer le traitement, l'indexation, la saisie et la mise èn disponibilité par le prêt entre bibliothèques. II est à noter que les différents comités provinciaux sont également soumis à cette règle du dépôt officiel. À titre d'exemple, tous les documents produits par le Comité de santé environnementale du Québec (avis, mémoires, ouvrages grand public, etc.) sont acheminés systématiquement à SANTÉCOM. Des ententes de dépôt officiel ont également été entérinées avec le Centre de recherche sur les services communautaires de l'Université Laval, la Fédération des CLSC du Québec (FCLSCQ), l'Association pour la santé publique du Québec (ASPQ) et le Groupe de recherche interdisciplinaire en santé (GRIS) de l'Université de Montréal.

Par ailleurs, plusieurs auteurs et/ou organismes collaborateurs du réseau de santé publique font parvenir leurs publications à SANTÉCOM sans même qu'une entente de dépôt ne soit conclue. Nous ne pouvons qu'encourager ces initiatives. La concentration et la recension des diverses publications au sein d'une seule source bibliographique présente un double avantage. D'une part, elle focalise la recherche documentaire en un point précis, ce qui permet de sauver un temps précieux, inévitable dans un contexte de dispersement. Ensuite, elle assure une plus grande visibilité, donc un plus grand lectorat aux auteurs, et en bout de ligne de meilleures retombées, aux recherches et programmes développés par les professionnels de la santé publique.

Bref, la pertinence d'une telle formule se trouvait encore renforcée en 1990 par l'orientation générale de la réforme du système de santé qui visait une meilleure efficience des services à tous les niveaux du système.

\section{Positionnementparrapportà d'autres banques de données}

Ni le ministère de la Santé et des Services sociaux du Québec, ni Santé Canada, ni même d'autres provinces canadiennes ne produisent de banque de données bibliographiques accessibles. dans le domaine de la santé publique. SANTÉCOM ne peut donc engendrer de redondance par rapport à d'autres fichiers nationaux abordant des thématiques similaires. Quant à MEDLINE - banque de données bibliographiques américaine, produite par la NATIONAL LIBRARY OF MEDICINE, dont la valeur est reconnue internationalement - elle dépouille et indexe systématiquement environ 3600 revues internationales. De plus, elle est mise à jour hebdomadairement. Elle est donc l'outil tout désigné pour parfaire les connaissances médicales et paramédicales. La mise à jour, le développement et la consultation de SANTÉCOM se fait donc en parallèle et non en concurrence avec les banques de données internationales que nous devrons toujours consulter pour nous maintenir à la fine pointe de l'information.

Du côté de la francophonie européenne, les banques de données bibliographiques spécialisées dans le domaine de la santé publique ne sont pas encore diffusées en ligne et n'offrent pas d'accès direct aux usagers, à une exception près: l'École nationale de la Santé publique de Rennes, qui encadre le développement de la banque de données Santé publique. Celle-ci regroupe un réseau de quatorze producteurs appelés à verser régulièrement leurs données dans la banque. Cette banque de données compte maintenant 41800 notices. Elle semble rencontrer certains problèmes de normalisation et de cohérence de l'indexâtion, ce qui entrave le repérage des données. Elle est maintenant diffusée à large échelle depuis 1994 , sur le MINITEL.

En Belgique, RÉSODOC, un collectif d'une quinzaine de partenaires producteurs de données, contribue depuis 1987 à l'élaboration d'une banque de données en santé publique. Elle contient aujourd'hui près de 8000 notices. Ce réseau a pour point d'ancrage l'Université catholique de Louvain. En Suisse, ce genre d'outil documentaire n'est pas encore disponible.

\section{Bilan rétrospectif: 1986-1995}

\section{Gestion du dossier}

SANTÉCOM est gérée de façon entièrement centralisée à partir du DSC Charles-LeMoyne, devenu depuis 1993, la DRSP de la Montérégie. L'élaboration du projet initial et le volet de "dépôt officiel», sa promotion auprès des autorités gouvernementales compétentes, son implantation et son développement, toutes ces étapes ont été réalisées sous l'initiative et la responsabilité de la bibliothécaire, responsable du Centre de documentation du DSC Charles-LeMoyne, assistée par une agente de bureau.

\section{Diffusion de la banque}

La banque de données SANTÉCOM a été diffusée, depuis sa création à la fin de 1986 jusqu'au printemps 1989, par la firme IST-INFORMATHĖQUE. Depuis décembre 
1989, la firme Services documentaires Multimedia (SDM) a pris le relais. On peut avoir accès à SANTÉCOM par télécommunication, soit directement chez SDM inc. (sans frais de télécommunication pour les usagers de la région de Montréal), soit par les réseaux DATAPAC, EDUPAC, INFOPUQ ou LE RÉSO (INET 2000) de Bell Canada. Dans tous les cas, le tarif est de $35 \$$ l'heure pour les membres du réseau de la santé et de $65 \$$ l'heure pour les autres. Les personnes ou les organismes qui ne disposent pas des ressources techniques requises pour effectuer une interrogation en direct, peuvent demander par téléphone à SDM inc. d'effectuer des recherches bibliographiques précises dans la banque de données SANTÉCOM: la tarification est alors établie en fonction du nombre de notices imprimées.

\section{Liste de descripteurs en santé publique}

La liste des descripteurs en langue française utilisés dans SANTÉCOM est une production maison. Les mises à jour annuelles tentent de suivre l'évolution du secteur de la santé publique au Québec. SANTÉCOM comporte une série de renvois Voir et Voir aussi, qui permettent un raffinement supplémentaire. La liste est publiée localement et est diffusée aux coûts d'impression (10 \$). II serait sans doute fort intéressant de systématiser cette liste de base pour en constituer un véritable thésaurus. Les ressources disponibles n'ont malheureusement pas permis de pousser les travaux dans cette direction.

\section{Les usagers}

Les usagers de SANTÉCOM en accès direct se concentrent principalement dans les secteurs public et parapublic, au sein d'organismes «bien nantis» au niveau documentaire. Ce sont, par exemple, des services documentaires bien développés avec des professionnels attitrés au service de référence et de téléréférence, des infrastructures informatiques complètes pour l'interrogation en direct et des serveurs de bases de données pertinentes. Une partie des usagers du réseau des DRSP ou des CLSC ne bénéficient pas de tels services. Ils utilisent plutôt le service de référence téléphonique offert par SDM inc. Quant aux usagers du centre de docu- mentation de la DRSP de la Montérégie, ils peuvent l'interroger sur place, et sans intermédiaire, grâce au logiciel NATUREL, d'une grande convivialité.

\section{Promotion de la banque}

Depuis sa mise en disponibilité en accès direct, la promotion de la banque de données a été assurée par le DSC Charles-LeMoyne, en collaboration avec ses partenaires successifs, IST et SDM inc.: production de dépliants promotionnels, participation à des congrès de l'AHQ, de la Fédération des CLSC du Québec, de I'ACFAS, etc. Cette réalisation a aussi bénéficié du soutien officiel du Comité de coordination de la santé communautaire de l'AHQ qui mandatait en 1987, le DSC Charles-LeMoyne comme dépositaire officiel des publications du réseau des DSC, et qui renouvelait son appui à la fin 1989 , en contribuant financièrement à la promotion de la banque. De même, les activités de représentation faites en Europe francophone au sein du Réseau francophone international en promotion de la santé (RÉFIPS) en 1993 et en 1994 ont été assumées par la DRSP de la Montérégie en collaboration avec le ministère de la Santé et des Services sociaux et l'Association pour la santé publique du Québec.

\section{Financement}

Depuis le début, le financement de ce projet a été entièrement assumé par le DSC Charles-LeMoyne, à même son budget de fonctionnement. La participation du réseau de santé publique se limite au dépôt officiel des publications et à quelques mises de fonds ponctuelles autour d'activités promotionnelles. II est à noter que la mise en disponibilité d'une banque de données par un serveur engendre des coûts que les redevances perçues auprès des utilisateurs ne compensent pas.

\section{Perspectives de développement}

La restructuration organisationnelle du secteur de la santé publique amorcée en 1993 n'est pas encore achevée. Mais plusieurs établissements ont entrepris de rationaliser leurs opérations. Les documentalistes du réseau sont déjà interpellés par ces réorganisations. II semble que la tendance soit de privilégier des centres de documentation moins nombreux mais plus étoffés ou semi-spécialisés. Quant à la DRSP de la Montérégie, elle continuera, en parallèle à la réorganisation du soutien documentaire dans sa région, de développer la banque de données SANTÉCOM. Elle tendra, entre autres, à élargir le dépôt officiel des publications afin d'atteindre plus systématiquement les partenaires immédiats de la santé publique. Le renforcement du volet international sur la base d'une collaboration bien entamée avec la France, la Belgique et la Suisse est également prévisible.

\section{Conclusion}

Lorsqu'on compare le réseau de santé publique québécois et les autres spécialités médicales ou paramédicales de même que les services qui les supportent, on constate facilement la «jeunesse» de notre réseau. De plus, ressortent l'éclatement et la multiplicité des points de contact que ce réseau engendre dans à peu près toutes les sphères de l'activité humaine.Si l'on devait représenter cette situation graphiquement, avec en ordonnée les années de «pratique» du réseau et en abscisse, les dossiers traités et leurs composantes intersectorielles, nous obtiendrions une série de courbes parallèles ascendantes qui témoigneraient de l'essor et de la diversité de notre secteur de recherche et d'intervention depuis vingt ans. La réforme actuelle des services de santé vise entre autres à resserrer davantage ce schéma pour favoriser le rapprochement, voire le croisement des différentes courbes.

Les centres de documentation se sont également développés de façon parallèle et selon une courbe ascendante au cours des dix dernières années. Cette même réforme les oblige à un resserrement et parfois à un recentrage thématique, afin que chaque centre désigné devienne semi-spécialisé. Le but évident consiste à éliminer certaines duplications qui plaçaient ces centres en position concurrentielle et à développer des expertises particulières, pour favoriser les échanges et l'exploitation des potentialités d'un véritable réseau documentaire. 


\section{Bibliographie}

Catalogue collectifdes périodiques dans les bibliothèques de la santé du Québec. Sources d'information québécoises, 3, Montréal: ASTED, 1994. $310 \mathrm{p}$.

Duplessis, P. et al. Santé publique et pays industrialisés. Québec: Les publications du Québec, 1989. $424 \mathrm{p}$.

EDUQ; bibliographie analytique surl'éducation au Québec 12(Printemps 1987).

Québec. Ministère de la santé et des services sociaux. La politique de santé et de bien-être. Québec, $1993.192 \mathrm{p}$.

Québec. Ministère de la santé et des services sociaux. Uneréforme axée sur le citoyen. Québec, 1990. 91 p. +annexes.

Répertoire des centres de documentation de la santé duQuébec. Sources d'information québécoises, 2, Montréal: ASTED, 1992. 154 p.

Rochon, Jean. «La santé communautaire dansle système régional de services de santé et de services sociaux.» In Annuaire du Québec, 1975-76. Québec, 1977,2e partie.

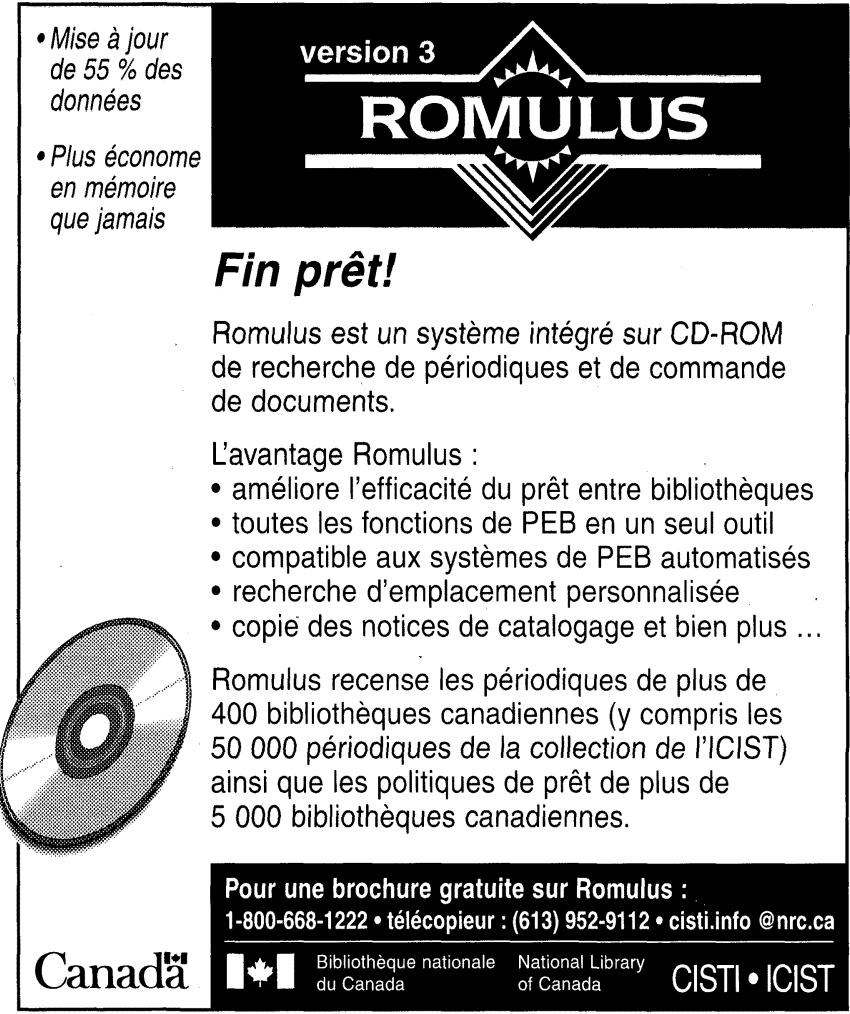

Documentation et bibliothèque $37 / 8 \times 91 / 2$

\section{La Bibliothèque québécoise}

\section{La plus grande source d'informations sur la société québécoise ... sur CD-ROM!}

Accès direct à plus de 510000 références bibliographiques: grande diversité de publications sur une période de plus de 12 ans et couvrant tous les sujets d'importance tels actualité, santé, affaires, communications, environnement, publications gouvernementales.

Outil de travail bibliographique: les documents repérés peuvent être imprimés selon une variété de formats ou transférés dans d'autres bases privées.

Repérage aisé, rapide et précis: I'interrogation s'effectue avec une version allégée de notre logiciel EdiBase avec menus déroulants à l'écran, questions en langage naturel ou avec opérateurs de recherche, index de recherche multiples, tri par ordre de pertinence, etc.

Bases: Index de l'actualité, Index des affaires, Index de la santé et des services sociaux; bibliographie sur les communications au Québec, sur l'environnement et l'aménagement du territoire québécois; publications gouvernementales québécoises.
801, rue Sherbrooke Est, bureau 615 Montréal (Québec) Canada H2L 1 K7 Téléphone : (514) 5247722 Télécopieur : 514 5245441 